\title{
Reliability of Mechanical and EMG Variables Assessed During Concentric Bench Press Exercise Against Different Submaximal Loads
}

\author{
Juan Manuel Yáñez García ${ }^{1}$, David Rodríguez Rosell*1, Ricardo Mora Custodio¹ , Antonio G Ravelo García² , Juan Ribas \\ Serna $^{3}$ and Juan José González Badillo ${ }^{1}$ \\ ${ }^{1}$ Physical Performance \& Sports Research Center, Pablo de Olavide University, Seville, Spain \\ ${ }^{2}$ Institute for Technological Development and Innovation in Communications, Universidad de Las Palmas de Gran Canaria, Spain \\ ${ }^{3}$ Department of Medical Physiology and Biophysics, University of Seville, Spain
}

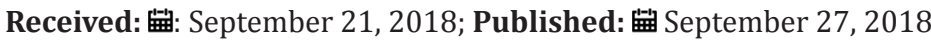

*Corresponding author: David Rodríguez Rosell, Physical Performance \& Sports Research Center, Pablo de Olavide University, Seville, Spain

\section{Abstract}

Purpose: To analyze the intrasession reliability of different kinetic, kinematic and surface electromyography (EMG) variables obtained during concentric phase of the bench press exercise against four different submaximal loads: 50\%,60\%,70\% and 80\% 1RM.

Methods: Twelve physical active men performed four testing sessions (one with each load magnitude) in a random order and separated by a time period of 3-4 days. In each session, participants were required to perform 3 sets of 2 repetitions each (3-min interset rests). The fastest repetition of each set was considered for subsequent reliability analysis. All data were analyzed using the intraclass correlation coefficient (ICC) and the coefficient of variation (CV).

Results: Variables related to force, velocity, power and acceleration presented high reliability scores (ICC: 0.920-0.997, CV: 1.22-8.87\%) against all load magnitudes used. The maximal rate of force development (MRFD) showed a high reproducibility, whereas the time taken to achieve the MRFD, time needed to attain different fractions of peak force and RFD quantify in different time bands $(0-25,0-50,0-75 \mathrm{~ms})$ resulted in low to high relative reliability (ICC: $0.550-0.978$ ) and moderate to poor absolute reliability (CV: 11.60-52.68\%). Absolute (CV: 3.59-57.52\%) and relative (ICC: 0.703-0.995) reproducibility for EMG variables related to signal amplitude and frequency of the power spectrum was fair to good, independent of load magnitude used and muscle assessed.

Conclusion: Overall, these results suggest that the reliability of the mechanical and EMG measures varied depending on the variable used to assess the performance during a concentric BP exercise.

Keywords: Force-Time Curve; Reproducibility; Power Spectrum EMG Variables; Dynamic Muscle Activation; Rate of Force Development

Abbreviations: 1RM: One-repetition maximum; ANOVA: Analysis of variance; BP: The bench press (BP) exercise; CI: Confidence interval; CV: Coefficient of variation; EMG: Electromyography; FInsm5: Dimitrov index; Fmax: Maximal frequency; Fmean: Mean frequency; Fmed: Median frequency; ICC: Intraclass correlation coefficient; iEMG: Integrated EMG; MPF: Mean propulsive force; MPV: Mean propulsive velocity; MRFD: Maximal RFD; MV: Mean velocity; PA: Peak acceleration; PF: Peak force; PM: Pectoralis major; PP: Peak power; PV: Peak velocity; RFD: Rate of force development; RFDAV: Average rate of force development; RMS: Root mean square; rRFD: RFD normalized relative to maximum peak force; SD: Standard deviations; SEM: Standard error of measurement; T_MRFD: Time from force onset to the instant of reaching MRFD T25\%, 50\%, 75\% and $100 \%$ Time taken to the attainment of $25 \%, 50 \%, 75 \%$ and $100 \%$ of the PF; TB: Triceps brachii

\section{Introduction}

The assessment of specific neuromuscular performance qualities provides relevant insight into the training status of an individual in relation to the sport requirements. This information is crucial for detecting potential deficiencies in strength and power capacities and, therefore, for designing individualized resistance training programs aimed at improving physical performance [1]
Skeletal muscle function can be evaluated using a wide variety of tests, which should provide reliable, valid, sensitive and specific measures to obtain a correct strength diagnosis $[2,3]$. Therefore, it would seem logical for coaches, strength and physical conditioning professionals and sport scientists to properly assess the reliability of different mechanical and physiological variables related to strength and power qualities [1]. 
The bench press (BP) exercise is a multi-joint movement beneficial to many functional activities and sporting movements and it is considered an important training exercise in many strength and conditioning programs in different sports [4]. For this reason, the BP exercise is usually used as a simple test for assessing the ability to produce force, velocity and power output in the upper limbs [4,5]. Some studies [5-9] have focused on analyzing the reliability of certain specific mechanical variables during dynamic BP exercise, such as peak force (PF) [5], mean propulsive velocity (MPV) $[7,10]$, mean velocity (MV) $[8,10]$, peak velocity (PV) $[5,10]$, peak power (PP) [5,9], and maximal rate of force development (MRFD) [5,6], showing moderate to high reliability scores depending on the analyzed variable. Despite these results, further investigation is required to assess the absolute and relative reliability of relevant kinetic and kinematic variables in the BP exercise.

In addition to mechanical variables, other physiological variables are usually assessed to analyze muscle performance. Surface electromyography (EMG) provides a non-invasive, objective method for measuring electrophysiological changes and assessing the neuromuscular function occurring during muscular contraction [11]. Thus, an improved understanding of the reliability of different EMG variables is warranted. Most studies examining the reliability of EMG variables have been limited to slow, controlled tasks, including gait analysis [12], isometric contractions $[13,14]$ or isokinetic dynamometry of single-joint exercises $[15,16]$, while few studies have analyzed the reliability of EMG variables during functional, dynamic, multi-joint exercises executing the movement at maximal intended velocity [11,17-19]. In these studies, EMG was measured during jump [17-19] or squat exercises [11], with reliability scores showing a wide variability depending on the exercise, variable and load magnitude used. However, we have no knowledge of any studies analyzing the reproducibility of EMG variables in upper-limb exercises such as concentric BP exercise. In addition, the variables analyzed in previous studies [11,17-19] were the root mean square (RMS), median frequency (Fmed) and integrated EMG (iEMG), whereas the reliability of other important EMG variables remains unanalyzed.

There exist several methodological factors that could impact on the reliability of the mechanical and EMG variables during BP assessments, including the type of muscle contraction $[6,20]$, determination of the signal (force or EMG) onset [21-23], sample sizes [20], population studied [2], contraction level and time intervals between measurements [20]. Another important factor influencing reliability during the BP exercise may be the method used to analyze the various force-time curve and EMG variables. Specifically, there are several methods reported in the literature that can be used for calculating the RFD from an isometric or dynamic force-time curve $[24,25]$. For example, it appears that the use of shorter time intervals in the calculations of RFD appears to considerably reduce reliability $[21,26]$. However, to the best of our knowledge, there are no studies analyzing the effect of sampling time interval on the reliability of EMG variables. On the other hand, when a dynamic test is conducted, the reliability of different kinetic, kinematic and EMG variables appears to also be affected by the magnitude of the external load [11,27]. Studies reporting the reliability of different mechanical variables across different loads during the BP exercise are scarce [6-9] and no differences in reliability scores for MPV, PV, PP and MRFD have been shown with regard to the load magnitude used. In contrast, a previous study [11] analyzing the reliability of EMG variables across different load magnitudes $(70 \%, 80 \%$ and $90 \% 1 \mathrm{RM})$ showed that the EMG variables assessed resulted in different absolute and relative reliability scores depending on the load magnitude used. However, this study was conducted using the squat exercise. Thus, further research appears to be required to clarify the impact of relative load on reliability in the different mechanical and EMG variables used as outcome measures for assessment during the BP exercise. Therefore, based on the above considerations, the aim of our study was to analyze the reliability of different kinetic, kinematic and EMG variables measured during the concentric phase of the BP exercise against four different submaximal loads (50\%, 60\%, 70\% and $80 \% 1 \mathrm{RM})$.

\section{Materials and Methods}

\section{Participants}

Twelve young and healthy men (age $24.6 \pm 3.9$ years, height $1.76 \pm 0.06 \mathrm{~m}$, body mass $75.1 \pm 6.3 \mathrm{~kg}$ ) volunteered to participate in this study. Their initial 1RM strength for the BP exercise was $77.5 \pm 10.8 \mathrm{~kg}(1.03 \pm 0.11$ normalized per $\mathrm{kg}$ of body mass). All participants were physically active sports science students with a RT experience ranging from 1 to 3 years (1-3 sessions/week) and were accustomed to performing the BP with correct technique. No physical limitations, health problems or musculoskeletal injuries that could affect testing were reported. None of the participants were taking drugs, medications or substances expected to affect physical performance or hormonal balance. The study was conducted according to the Declaration of Helsinki and was approved by the Research Ethics Committee of Pablo de Olavide University. After being informed of the purpose and experimental procedures, the participants signed a written informed consent form prior to participation.

\section{Study Design}

A descriptive design was used to assess the absolute and relative reliability of different mechanical and EMG variables during concentric BP exercise against four load magnitudes: 50\%, 60\%, $70 \%$ and $80 \% 1 \mathrm{RM}$. To address this, twelve physically active men performed five testing sessions in the following order: 1) an initial test with increasing loads for the individual determination of 1RM strength and full load-velocity relationship in the BP exercise; and 2) four testing sessions with different relative loads $(50 \%, 60 \%$, $70 \%$ and $80 \% 1 \mathrm{RM}$ ), in which kinetic and kinematics variables and EMG signals from pectoralis major (PM) and triceps brachii (TB) were recorded. These 4 testing sessions were randomized for each participant and were separated by a time period of 3-4 days. Sessions were performed at the same time of day $( \pm 1 \mathrm{~h})$ for each participant and under similar environmental conditions $\left(\sim 20-22^{\circ} \mathrm{C}\right.$ and 55\%-65\% humidity). Testing was performed using a Smith 
machine (Multipower Fitness Line, Peroga, Spain). During the week preceding this study, two preliminary familiarization sessions were undertaken with the purpose of emphasizing the proper execution technique for the BP exercise.

\section{Testing Procedure}

Isoinertial Progressive Loading Test in the BP Exercise: Testing was performed using a Smith machine. Participants laid supine on a flat bench, with their feet resting flat on the floor and hands placed on the bar slightly wider $(2-3 \mathrm{~cm})$ than shoulder width. The position on the bench was carefully adjusted so that the vertical projection of the bar corresponded with each participant's intermammary line. The individual positions on the bench as well as grip widths were measured so that they could be reproduced on every lift. Participants were not allowed to bounce the bar off their chests or raise the shoulders or trunk off the bench. Two telescopic bar holders with a precision scale were placed at the left and right sides of the Smith machine in order to: (i) precisely replicate the individual eccentric range of movement between trials; and (ii) impose a pause or delay between the eccentric and concentric phases of the BP exercise. The bar holders were positioned so that the bar stopped $\sim 1 \mathrm{~cm}$ above each participant's chest. After lowering the bar at a controlled mean eccentric velocity $(\sim 0.30$ $0.50 \mathrm{~m} \cdot \mathrm{s}^{-1}$ ), participants stopped for $\sim 1.5 \mathrm{~s}$ at the bar holders (momentarily releasing the weight but keeping contact with the bar), and thereafter they performed a purely concentric push at maximal intended velocity. This momentary pause between phases was imposed in order to minimize the contribution of the rebound effect and allow for more reliable, consistent measures [7]. Each participant was carefully instructed to always perform the concentric phase of each repetition in an explosive manner, exploding the bar off the chest as fast as possible upon hearing the 'go!' command from a researcher. Warm-up consisted of $5 \mathrm{~min}$ of joint mobilization exercises, followed by 2 sets of 8 and 6 repetitions ( $3 \mathrm{~min}$ rest) with loads of 20 and $30 \mathrm{~kg}$, respectively. The initial load was set at $20 \mathrm{~kg}$ for all participants and was gradually increased in 10-kg increments until the attained MPV was lower than $0.4 \mathrm{~m} \cdot \mathrm{s}^{-1}$. Thereafter, the load was individually adjusted with smaller increments ( 5 down to $1 \mathrm{~kg}$ ) so that $1 \mathrm{RM}$ could be precisely determined. The heaviest load that each participant could properly lift while completing full range of motion was considered to be his 1RM. Two trained spotters were present when high loads were lifted to ensure safety. Three attempts were executed for light (MPV $\left.>0.95 \mathrm{~m} \cdot \mathrm{s}^{-1}\right)$, two for medium $\left(0.95 \mathrm{~m} \cdot \mathrm{s}^{-1}>\mathrm{MPV}>0.55 \mathrm{~m} \cdot \mathrm{s}^{-1}\right)$, and only one for the heaviest (MPV $<0.55 \mathrm{~m} \cdot \mathrm{s}^{-1}$ ) loads. Interset rests ranged from 3 (light) to $5 \mathrm{~min}$ (heavy loads).

Reliability Test in the BP Exercise: Each participant performed 4 reliability tests every 3-4 days with different submaximal loads: $50 \%, 60 \%, 70 \%$, and $80 \% 1 \mathrm{RM}$, respectively. During each testing session, participants were required to perform 3 sets of 2 repetitions each (3-min interset rests). The best repetition of each set, according to the criteria of fastest MPV, was considered for subsequent reliability analysis. Before starting each reliability testing session (50-80\% 1RM, in 10\% increments), adjustments in the load $(\mathrm{kg})$ to be used were made when needed so that the velocity of the first repetition matched the specified target MPV corresponding to each load. These target MPV to be attained in the first (usually the fastest) repetition of the set in each session were the following: $\sim 0.93 \mathrm{~m} \cdot \mathrm{s}^{-1}(50 \% 1 \mathrm{RM}), \sim 0.79 \mathrm{~m} \cdot \mathrm{s}^{-1}(60 \% 1 \mathrm{RM})$, $\sim 0.62 \mathrm{~m} \cdot \mathrm{s}^{-1}(70 \% 1 \mathrm{RM})$, and $\sim 0.47 \mathrm{~m} \cdot \mathrm{s}^{-1}(80 \% 1 \mathrm{RM})$ [28]. The absolute load (kg) for each participant was individually adjusted to match the velocity associated $\left( \pm 0.02 \mathrm{~m} \cdot \mathrm{s}^{-1}\right)$ with the $\% 1 \mathrm{RM}$ intended for each session. During each test, the participants were required to move the bar as fast as possible during the concentric phase of each repetition. As done for the isoinertial progressive loading test, the participants were required to perform each repetition descending the bar in a controlled manner and maintain a static position during $\sim 1.5 \mathrm{~s}$ at the end of the eccentric phase (on the bar holders) before lifting the bar as fast as possible upon hearing a verbal command. Participants were asked to avoid any countermovement before force onset. Warm-up consisted of $5 \mathrm{~min}$ of joint mobilization exercises, followed by three or four sets with progressive loads (3-min rest).

\section{EMG Recording}

Surface EMG during the BP exercise was recorded from the PM and TB of the right side using pairs of bipolar surface electrodes (Blue Sensor N-00-S, Medicotest) with a distance between the electrode's centers of $22 \mathrm{~mm}$. After careful preparation of the skin by shaving and cleaning with alcohol, surface electrodes were placed over the belly of the muscle, parallel to the presumed orientation of the muscle fibers of PM and TB, according to SENIAM (Surface EMG for Non-Invasive Assessment of Muscles) guidelines [29]. All electrode positions were carefully measured in each participant to ensure identical recording sites were used in all testing sessions. The reference electrode was placed on the anterior superior iliac spine on the same side. Skin-electrode impedance was assessed on each occasion and maintained at a consistent level (within $0.5 \mathrm{M} \Omega$ ) within individuals and at a value $<5 \mathrm{M} \Omega$ for all participants. EMG signals were synchronized with kinetic and kinematic data by recording at $1000 \mathrm{~Hz}$ with the same analogue-to-digital converter and PC as the kinetic and kinematic signals. During off-line analysis the signals were band-pass filtered in both directions between 6 and $500 \mathrm{~Hz}$ using a second order Butterworth digital filter. The parameters analyzed in the present study corresponded to the first $500 \mathrm{~ms}$ of the concentric phase of the BP exercise in both PM and TB muscles.

\section{Measurement Equipment and Data Acquisition}

A portable force platform (T-Force Dynamic Measurement System, Ergotech, Murcia, Spain), which was synchronized with a linear velocity transducer (T-Force Dynamic Measurement System, Ergotech, Murcia, Spain), was used for measuring the relevant kinetic and kinematic parameters of every repetition. Both devices the force platform and linear velocity transducer were connected to a board computer and recorded data at a sample rate of $1,000 \mathrm{~Hz}$ and was used for measuring the force-time and velocity-time curves during the concentric phase for each repetition performed throughout the whole range of motion. The mean propulsive 
force (MPF) was defined as the force applied from force onset to the moment which the measured acceleration (a) is greater than acceleration due to gravity (i.e., a $\geq-9.81 \mathrm{~m} \cdot \mathrm{s}^{-2}$ ), whereas the peak force (PF) was defined as the maximum ground reaction force achieved during the concentric phase. The time taken from force onset to the attainment of different fractions (e.g. 25\%, 50\%, 75\% and $100 \%$ ) of the PF was also analyzed. Instantaneous power was calculated from the product of velocity captured with the linear transducer and the ground reaction force measured by the portable force platform during each millisecond, although only peak power (PP) was used for the subsequent analysis. The RFD was analyzed with the different methods that have been reported in the scientific literature. The maximal rate of force development (MRFD) was calculated as the peak slope of the force-time curve during different time intervals $(4,10,20,40,50$ and $60 \mathrm{~ms})$ sampling windows $[24,26]$. The time taken from force onset to the moment at which MRFD is reached was also analyzed (T_MRFD). RFD was also derived as the average slope of the force-time curve over time intervals of 0-25 (RFD0-25), 0-50 (RFD0-50), 0-75ms (RFD0-75) relative to the onset of contraction, in absolute terms $\left(\mathrm{N} \cdot \mathrm{s}^{-1}\right)[24,30]$ and when the force values were normalized ( $\mathrm{rRFD}$ ) relative to maximum force (expressed as $\% \mathrm{MF} \cdot \mathrm{S}^{-1}$ ) [30]. The RFD was also calculated as the time taken from the beginning of force production to the attainment of different fractions of the maximal voluntary contraction (T25\%, T50\%, T75\% and T100\%) [13]. Finally, RFD was quantified as the ratio between the peak force when a plateau in the force-time curve is achieved and the time it took to reach the given force level (RFDAV) [24]. The linear velocity transducer was used for MPV, peak velocity (PV) and peak acceleration (PA) measuring.

EMG signals were synchronized with kinetic and kinematic data by recording at $1000 \mathrm{~Hz}$ with the same analogue to digital converter and PC as the kinetic and kinematic signals. EMG data were collected using LabChart software version 7.0 (National Instruments Corporation. Austin, TX, USA), and data analysis was performed off-line using the MATLAB 2011a software environment (The MathWorks Inc., Natick, Massachusetts, USA). EMG and force signal onsets were identified with a standardized systematic protocol of visual identification using a standard method by two trained experienced investigators described in previous studies $[22,23]$. The following EMG parameters were calculated:

\section{a) Root mean square (RMS):}

$$
R M S=\sqrt{\frac{1}{N} \sum_{i=1}^{N} x_{i}^{2}}
$$

b) EMG integrated:

$$
i E M G=\int_{t_{1}}^{t_{2}}|S(t)| d t m V
$$

c) Mean frequency (Fmean):

d) Median frequency (Fmed):

$$
\text { Fmean }=\frac{m_{1}}{m_{0}}=\frac{\int_{0}^{f} f S(f) d f}{\int_{0}^{f} S(f) d f}
$$

$$
\int_{0}^{\text {fmed }} S(f) d f=\int_{f \text { med }}^{\infty} S(f) d f
$$

\section{e) Maximal frequency (Fmax)}

f) The new spectral parameter proposed by Dimitrov (FInsm5) [31]:

$$
f \operatorname{Insm} 5=\frac{\int_{f_{1}}^{f_{2}} f^{-1} \cdot P S(f) \cdot d f}{\int_{f_{1}}^{f_{2}} f^{5} \cdot P S(f) \cdot d f}
$$

\section{Statistical Analyses}

Standard statistical methods were used for the calculation of means and standard deviations (SD). A one-way repeated measure analysis of variance (ANOVA) was used to detect differences in mechanical and EMG variables between the three attempts against all four load magnitudes [2]. Absolute and relative reliability was assessed for each variable. A one-way random effects model (model 2,1) ICC with absolute agreement was used to determine relative reliability $[2,32]$. The size of the correlation was evaluated as follows: $r<0.7$ low; $0.7 \leq r<0.9$ moderate and $r \geq 0.9$ high $[2,32]$. Absolute reliability was reported using the standard error of measurement (SEM $=\sqrt[2]{\text { ErrorMean Square }}$ ) [32]. The SEM values were expressed as a percentage of their respective means through the CV $[2,3]$. A CV of $\leq 10 \%$ was set as the criterion to declare a variable as reliable $[2,3,32]$. Statistical significance was set at $p<$ 0.05. All data analyses were performed using SPSS (V18.0; SPSS, Inc., Chicago, Illinois, USA).

\section{Results}

Data for all variables analyzed were homogeneous and normally distributed ( $p>0.05$ ). Descriptive characteristics of the load actually performed in all four testing sessions are reported in Table 1. No significant differences were found between the expected or targeted MVP values and the average MPV values of each attempt at any of the load magnitudes used. In addition, oneway repeated measures ANOVA revealed no significant differences between attempts in any mechanical and EMG variable for any load intensity used.

Table 1: Descriptive data of the load magnitudes used in each testing session.

\begin{tabular}{|c|c|c|c|c|c|c|c|}
\hline Scheduled & \multicolumn{5}{|c|}{ Actually Performed } \\
\hline \multirow{2}{*}{ \%1RM (associated MPV) } & \multicolumn{3}{|c|}{ MVP $\left(\mathbf{m} \cdot \mathbf{s}^{-1}\right)$} & & \multirow{2}{*}{ \%1RM } \\
\cline { 2 - 7 } & Attempt 1 & Attempt 2 & Attempt 3 & Attempt 1 & Attempt 2 & Attempt 3 & \\
\hline $\mathbf{5 0 \%}\left(\sim 0.93 \mathrm{~m} \cdot \mathrm{s}^{-1}\right)$ & $0.94 \pm 0.01$ & $0.93 \pm 0.01$ & $0.94 \pm 0.01$ & $50.5 \pm 0.7$ & $51.1 \pm 0.8$ & $50.7 \pm 0.8$ & $37.5 \pm 5.7$ \\
\hline $\mathbf{6 0 \%}\left(\sim 0.79 \mathrm{~m} \cdot \mathrm{s}^{-1}\right)$ & $0.79 \pm 0.01$ & $0.78 \pm 0.01$ & $0.78 \pm 0.01$ & $59.6 \pm 0.7$ & $60.0 \pm 0.6$ & $59.8 \pm 0.8$ & $45.0 \pm 6.7$ \\
\hline $\mathbf{7 0 \%}\left(\sim 0.62 \mathrm{~m} \cdot \mathrm{s}^{-1}\right)$ & $0.62 \pm 0.01$ & $0.62 \pm 0.01$ & $0.62 \pm 0.01$ & $70.0 \pm 0.7$ & $70.2 \pm 0.6$ & $70.2 \pm 0.7$ & $54.6 \pm 7.6$ \\
\hline $\mathbf{8 0 \%}\left(\sim 0.47 \mathrm{~m} \cdot \mathrm{s}^{-1}\right)$ & $0.47 \pm 0.02$ & $0.47 \pm 0.02$ & $0.47 \pm 0.02$ & $79.5 \pm 1.0$ & $79.9 \pm 1.3$ & $79.5 \pm 1.2$ & $62.6 \pm 7.9$ \\
\hline
\end{tabular}

The values given are the means \pm SD. 1RM: One-repetition maximum; MPV: Mean propulsive velocity. 


\section{Reliability of Mechanical Variables}

The reliability of different kinetic and kinematic variables is displayed in Table 2, and the reliability of RFD variables is presented in Table 3. The variables PV, PA, PP, PF and MPF showed high reliability scores, with ICC values higher than 0.920 and CV values ranging from $1.64-8.87 \%$ (Table 2 ).

Table 2: Reliability (ICC and CV) for different mechanical variables assessed during the BP exercise against all four load magnitudes.

\begin{tabular}{|c|c|c|c|c|c|c|c|c|}
\hline \multirow[b]{2}{*}{ Variables } & \multicolumn{2}{|l|}{$50 \% 1 R M$} & \multicolumn{2}{|l|}{$60 \% 1 \mathrm{RM}$} & \multicolumn{2}{|l|}{$70 \% 1 \mathrm{RM}$} & \multicolumn{2}{|l|}{$80 \% 1 R M$} \\
\hline & ICC (CI: 95\%) & CV & ICC (CI: 95\%) & CV & ICC (CI: 95\%) & CV & ICC (CI: 95\%) & CV \\
\hline $\operatorname{MPV}\left(\mathrm{m} \cdot \mathrm{s}^{-1}\right)$ & $0.098(-1.333-0.716)$ & 1.41 & $-0.026(-1.654-0.677)$ & 1.49 & $\begin{array}{c}0.532(-0.210- \\
0.853)\end{array}$ & 1.4 & $0.571(-0.425-0.875)$ & 2.9 \\
\hline $\operatorname{MV}\left(\mathrm{m} \cdot \mathrm{s}^{-1}\right)$ & $0.631(0.044-0.884)$ & 1.22 & $0.629(0.040-0.883)$ & 1.46 & $0.662(0.125-0.893)$ & 1.33 & $0.633(-0.220-0.893)$ & 2.62 \\
\hline $\mathrm{PV}\left(\mathrm{m} \cdot \mathrm{s}^{-1}\right)$ & $0.950(0.871-0.984)$ & 1.64 & $0.951(0.873-0.985)$ & 1.71 & $0.957(0.889-0.986)$ & 1.89 & $0.927(0.759-0.979)$ & 2.45 \\
\hline $\mathrm{PA}\left(\mathrm{m} \cdot \mathrm{s}^{-2}\right)$ & $0.954(0.880-0.985)$ & 3.66 & $0.951(0.873-0.985)$ & 3.86 & $0.930(0.819-0.978)$ & 5.61 & $0.925(0.751-0.978)$ & 6.24 \\
\hline PP (w) & $0.985(0.957-0.996)$ & 4.3 & $0.985(0.962-0.995)$ & 4.93 & $0.958(0.880-0.988)$ & 8.67 & $0.920(0.729-0.963)$ & 8.87 \\
\hline $\mathrm{PF}(\mathrm{N})$ & $0.976(0.938-0.992)$ & 3.8 & $0.970(0.922-0.990)$ & 4.43 & $0.972(0.927-0.991)$ & 3.83 & $0.967(0.890-0.990)$ & 3.04 \\
\hline MPF (N) & $0.978(0.944-0.993)$ & 3.98 & $0.997(0.992-0.999)$ & 1.55 & $0.995(0.986-0.998)$ & 1.77 & $0.974(0.915-0.993)$ & 3.38 \\
\hline
\end{tabular}

1RM: One-Repetition Maximum; CCI: Intraclass Correlation Coefficient; CI: Confidence Interval; CV: Coefficient of Variation; MPV: Mean Propulsive Velocity; MV: Mean Velocity; PV: Peak Velocity; PA: Peak Acceleration; PP: Peak Power; PF: Peak Force; MPF: Mean Propulsive Force.

Regarding RFD variables (Table 3), the MRFD quantified during different time interval sampling windows $(4,10,20,40,50$ and $60 \mathrm{~ms}$ ) showed high ICC scores and low to moderate CV against all four load magnitudes used, except for MRFD 4, 10, 20, and 40ms sampling window at $80 \% 1 \mathrm{RM}$, where the ICC and CV values showed a moderate reproducibility. In addition, there was a slight tendency to find greater reliability as the sampling window used to calculate the MRFD grew larger. The variable T_MRFD showed a moderate to low reliability (CV: $13.49-29.74 \%$ ), regardless of the load magnitudes used or the sampling window taken to calculate the MRFD. In addition, with the exception of the RFD0-25 at 50\% 1RM (ICC: 0.55), absolute RFD at different time bands achieved moderate ICC scores (0.722 - 0.884) in all four load magnitudes under study (Table 3), although these variables showed a high intra-subject variability (CV: $18.36-52.68 \%$ ). There was a tendency to find greater ICC and lower CV values as the time interval taken to calculate the absolute RFD decreased. However, when the force values were normalized relative to maximum $P F$, both criteria used to assess reliability (ICC and CV) showed similar values for RFD measured in different time bands. In general, the time taken from the beginning of force production to the attainment of different fractions $(25,50,75$ and $100 \%)$ of the peak force presented moderate relative reliability (ICC: $0.633-0.978$ ) and low absolute reliability (CV: 11.60 - 22.18\%), without a clear difference between the four load magnitudes used (Table 3).

Table 3: Reliability (ICC and CV) for different variables used to quantify rate of force development during the BP exercise against all four load magnitudes.

\begin{tabular}{|c|c|c|c|c|c|c|c|c|}
\hline \multirow[b]{2}{*}{ Variables } & \multicolumn{2}{|l|}{$50 \% 1 \mathrm{RM}$} & \multicolumn{2}{|l|}{$60 \% 1 \mathrm{RM}$} & \multicolumn{2}{|l|}{$70 \% 1 \mathrm{RM}$} & \multicolumn{2}{|l|}{$80 \% 1 \mathrm{RM}$} \\
\hline & ICC (CI: 95\%) & CV & ICC (CI: 95\%) & CV & ICC (CI: 95\%) & CV & ICC (CI: 95\%) & CV \\
\hline $\mathrm{MRFD}_{4}\left(\mathrm{~N} \cdot \mathrm{s}^{-1}\right)$ & 0929060 & 896 & $3-0$ & 3.79 & $976(0.937-0.992)$ & 7.37 & $0.700(0.004-0.913)$ & 15.27 \\
\hline $\operatorname{MRFD}_{10}\left(\mathrm{~N} \cdot \mathrm{s}^{-1}\right)$ & $942(0.850-0.982)$ & 8.71 & $907(0.761-0.971)$ & 13.02 & $0.965(0.910-0.989)$ & 9.27 & $0.727(0.094-0.920)$ & 17 \\
\hline $\operatorname{MRFD}_{20}\left(\mathrm{~N} \cdot \mathrm{s}^{-1}\right)$ & $45(0.050-0.903)$ & 9.05 & $10(0.100-0.9 / 4)$ & 2.9 & $0.972(0.927-0.991)$ & 8.38 & $0.799(0.332-0.941)$ & 15.84 \\
\hline $\operatorname{MRFD}_{40}\left(\mathrm{~N} \cdot \mathrm{s}^{-1}\right)$ & $0.941(0.847-0.981)$ & 9.49 & $0.943(0.853-0.982)$ & 10.46 & $0.970(0.921-0.990)$ & 8.34 & $0.871(0.571-0.962)$ & 12.17 \\
\hline $\mathrm{MRFD}_{50}\left(\mathrm{~N} \cdot \mathrm{s}^{-1}\right)$ & $0.957(0.888-0.986)$ & 8. & c & 9.84 & ) & 7 & ) & 9.08 \\
\hline $\operatorname{MRFD}_{60}\left(\mathrm{~N} \cdot \mathrm{s}_{-1}\right)$ & $0.951(0.873-0.948)$ & 8.82 & $0.925(0.807-0.976)$ & 11.07 & $0.973(0.931-0.992)$ & 7.2 & $0.943(0.811-0.983)$ & 7.88 \\
\hline $\mathrm{T}_{-} \mathrm{MRFD}_{4}(\mathrm{~s})$ & $0.754(0.363-0.922)$ & 25.59 & $0.769(0.402-0.927)$ & 25.56 & $0.727(0.283-0.913)$ & 26.93 & $0.698(-0.003-0.912)$ & 23.69 \\
\hline $\mathrm{T}_{-}$MRFD10 (s) & $0.732(0.306-0.915)$ & 25.88 & $0.824(0.546-0.945)$ & 22.53 & $0.735(0.314-0.916)$ & 26.6 & $0.672(-0.089-0.904)$ & 25.1 \\
\hline $\mathrm{T}_{-} \mathrm{MRFD}_{20}(\mathrm{~s})$ & $0.585(-0.073-0.869)$ & 29.74 & $0030<0-05040)$ & 21.87 & $0.699(0.221-0.905)$ & 26.27 & $0.688(-0.036-0.909)$ & 24.51 \\
\hline $\mathrm{T}_{-} \mathrm{MRFD}_{40}(\mathrm{~s})$ & $0.735(0.315-0.916)$ & 22.68 & $0.863(0.645-0.957)$ & 20.27 & $0.697(0.216-0.904)$ & 27.26 & $0.735(0.121-0.923)$ & 22.03 \\
\hline $\mathrm{T}_{-} \mathrm{MRFD}_{50}(\mathrm{~s})$ & $0.672(0.152-0.897)$ & 22.81 & $0.856(0.627-0.955)$ & 19.39 & $0.748(0.349-0.921)$ & 25.5 & $0.922(0.740-0.977)$ & 13.49 \\
\hline $\mathrm{T}_{-} \mathrm{MRFD}_{60}(\mathrm{~s})$ & $0.708(0.244-0.908)$ & 20.31 & $0.870(0.663-0.959)$ & 16.69 & $0.760(0.379-0.924)$ & 25.92 & $0.929(0.763-0.979)$ & 13.81 \\
\hline
\end{tabular}




\begin{tabular}{|c|c|c|c|c|c|c|c|c|}
\hline $\mathrm{RFD}_{0-25}\left(\mathrm{~N} \cdot \mathrm{s}_{-1}\right)$ & $0.550(-0.164-0.858)$ & 43.53 & $0.724(0.286-0.913)$ & 52.68 & $0.813(0.517-0.941)$ & 40.18 & $0.746(0.039-0.936)$ & 37.82 \\
\hline $\mathrm{RFD}_{0-50}\left(\mathrm{~N} \cdot \mathrm{s}_{-1}\right)$ & $0.802(0.487-0.937)$ & 27.13 & $0.832(0.566-0.947)$ & 32.35 & $0.806(0.497-0.939)$ & 30.34 & $0.857(0.496-0.961)$ & 26.06 \\
\hline $\mathrm{RFD}_{0-75}\left(\mathrm{~N} \cdot \mathrm{s}_{-1}\right)$ & $0.856(0.627-0.955)$ & 21.17 & $0.879(0.686-0.962)$ & 21.89 & $0.722(0.281-0.912)$ & 26.37 & $0.884(0.592-0.968)$ & 18.36 \\
\hline $\mathrm{rRFD}_{0-25}\left(\mathrm{~N} \cdot \mathrm{s}_{-1}\right)$ & $0.883(0.696-0.963)$ & 28.14 & $0.889(0.714-0.965)$ & 28.94 & $0.913(0.765-0.974)$ & 39.06 & $0.918(0.712-0.978)$ & 26.88 \\
\hline $\mathrm{rRFD}_{0-50}\left(\mathrm{~N} \cdot \mathrm{s}_{-1}\right)$ & $0.803(0.492-0.938)$ & 23.99 & $0.873(0.672-0.960)$ & 24.93 & $0.903(0.750-0.970)$ & 26.87 & $0.866(0.527-0.963)$ & 26.99 \\
\hline $\mathrm{rRFD}_{0-75}\left(\mathrm{~N} \cdot \mathrm{s}_{-1}\right)$ & $0.792(0.461-0.934)$ & 20.13 & $0.878(0.686-0.962)$ & 18.45 & $0.825(0.548-0.945)$ & 24.25 & $0.864(0.520-0.963)$ & 19.58 \\
\hline $\mathrm{RFD}_{\mathrm{AV}}\left(\mathrm{N} \cdot \mathrm{s}_{-1}\right)$ & $0.928(0.814-0.977)$ & 16.53 & $0.818(0.529-0.943)$ & 19.53 & $0.843(0.577-0.954)$ & 17.74 & $0.847(0.492-0.955)$ & 14.36 \\
\hline $\mathrm{T}_{25 \%}(\mathrm{~s})$ & $0.707(0.208-0.913)$ & 22.18 & $0.881(0.692-0.962)$ & 16.9 & $0.808(0.482-0.943)$ & 21.06 & $0.897(0.638-0.972)$ & 18.39 \\
\hline $\mathrm{T} 50 \%(\mathrm{~s})$ & $0.693(0.207-0.903)$ & 19.22 & $0.880(0.689-0.962)$ & 14.66 & $0.771(0.408-0.928)$ & 19.33 & $0.936(0.786-0.981)$ & 11.6 \\
\hline $\mathrm{T} 75 \%(\mathrm{~s})$ & $0.633(0.051-0.884)$ & 19.03 & $0.882(0.695-0.963)$ & 12.06 & $0.811(0.511-0.940)$ & 16.08 & $0.931(0.772-0.980)$ & 11.76 \\
\hline $\mathrm{T} 100 \%(\mathrm{~s})$ & $0.978(0.942-0.993)$ & 14.94 & $0.825(0.528-0.948)$ & 15.4 & $0.869(0.661-0.959)$ & 15.75 & $0.915(0.717-0.975)$ & 12.47 \\
\hline
\end{tabular}

1RM: One-repetition maximum; ICC: Intraclass correlation coefficient; CI: Confidence interval; CV: Coefficient of variation; MRFD: Peak rate of force development during different time intervals sampling window $(4,10,20,40,50$ and 60ms); T_MRFD: Time from force onset to the instant of reaching MRFD; RFD: Average slope of the force-time curve over different time intervals (0-25, 0-50, $0-75 \mathrm{~ms})$ relative to the onset of contraction; rRFD: Average slope of the force-time curve over different time intervals $(0-25,0-50$, $0-75 \mathrm{~ms}$ ) when the force values were normalized relative to maximum peak force; RFDAV: Average rate of force development; T25\%, $50 \%, 75 \%$ and $100 \%$ : Time taken from the beginning of force production to the attainment of $25 \%, 50 \%, 75 \%$ and $100 \%$ of the peak force.

\section{Reliability of EMG Variables}

Absolute and relative reliability of the different EMG variables of the PM and TB muscles are displayed in Tables $4 \& 5$, respectively. The RMS, iEMG and WDT2 were the most reliable EMG variables in both the PM (ICC: $0.984-0.995$; CV: $8.03-13.65 \%$ ) and TB muscles (ICC: 0.893 - 0.985; CV: 10.73 - 16.66\%), with no differences between load magnitudes used. The EMG power spectrum variables (Fmed, Fmean and Fmax) presented moderate to high relative (CCI: $0.703-0.952$ ) and absolute reliability scores (CV: $3.60-17.17 \%$ ) for all four relative loads used (Tables 4,5). For both muscles and all four load magnitudes analyzed, the FInsm5 showed moderate to high ICC scores, whereas the CVs were high, with values ranging from $23.41-30.23 \%$ (Tables 4 \& 5).

Table 4: Reliability (ICC and CV) for different sEMG variables assessed in the pectoralis major muscle during the BP exercise against all four load magnitudes.

\begin{tabular}{|c|c|c|c|c|c|c|c|c|}
\hline \multirow[b]{2}{*}{ Variables } & \multicolumn{2}{|l|}{$50 \% 1 \mathrm{RM}$} & \multicolumn{2}{|l|}{$60 \% 1 \mathrm{RM}$} & \multicolumn{2}{|l|}{$70 \% 1 \mathrm{RM}$} & \multicolumn{2}{|l|}{$80 \%$ 1RM } \\
\hline & ICC (CI: 95\%) & CV & ICC (CI: 95\%) & CV & ICC (CI: 95\%) & $\mathrm{CV}$ & ICC (CI: 95\%) & CV \\
\hline RMS & $0.989(0.970-0.997)$ & 9.70 & $0.987(0.965-0.996)$ & 13.65 & $0.987(0.965-0.996)$ & 10.43 & $0.984(0.939-0.996)$ & 8.03 \\
\hline iEMG & $0.985(0.960-0.996)$ & 10.85 & $0.987(0.965-0.996)$ & 13.65 & $0.987(0.965-0.996)$ & 10.43 & $0.984(0.939-0.996)$ & 8.03 \\
\hline Fmed & $0.821(0.493-0.951)$ & 3.60 & $0.784(0.442-0.932)$ & 5.86 & $0.869(0.647-0.961)$ & 3.59 & $0.774(0.204-0.938)$ & 6.51 \\
\hline Fmean & $0.810(0.487-0.944)$ & 6.42 & $0.703(0.198-0.912)$ & 5.60 & $0.858(0.632-0.955)$ & 4.12 & $0.770(0.378-0.932)$ & 6.00 \\
\hline Fmax & $0.725(0.220-0.925)$ & 9.81 & $0.711(0.220-0.915)$ & 14.00 & $0.725(0.258-0.919)$ & 6.55 & $0.848(0.424-0.962)$ & 9.48 \\
\hline FInsm5 & $0.842(0.551-0.957)$ & 30.23 & $0.856(0.627-0.955)$ & 23.41 & $0.917(0.776-0.975)$ & 28.95 & $0.801(0.435-0.946)$ & 28.62 \\
\hline
\end{tabular}

1RM: One-Repetition Maximum; ICC: Intraclass Correlation Coefficient; CI: Confidence Interval; CV: Coefficient of Variation; EMG: Electromyography; RMS: Root Mean Square; IEMG: Integrated EMG; Fmed: Median Frequency; Fmean: Mean Frequency; Fmax: Maximal Frequency; Finsm5: The New Spectral Parameter Proposed by Dimitrov.

Table 5: Reliability (ICC and CV) for different sEMG variables assessed in the triceps brachii muscle during the BP exercise against all four load magnitudes.

\begin{tabular}{|c|c|c|c|c|c|c|c|c|}
\hline & \multicolumn{2}{|c|}{$\mathbf{5 0 \%}$ 1RM } & \multicolumn{2}{c|}{$\mathbf{6 0 \%}$ 1RM } & \multicolumn{2}{c|}{ 70\% 1RM } & \multicolumn{1}{c|}{$\mathbf{8 0 \%}$ 1RM } \\
\hline Variables & ICC (CI: 95\%) & CV & ICC (CI: 95\%) & CV & ICC (CI: 95\%) & CV & ICC (CI: 95\%) & CV \\
\hline RMS & $0.937(0.830-0.981)$ & 11.90 & $0.980(0.949-0.994)$ & 10.73 & $0.947(0.862-0.983)$ & 11.02 & $0.982(0.951-0.995)$ & 15.27 \\
\hline iEMG & $0.917(0.777-0.976)$ & 12.91 & $0.980(0.949-0.994)$ & 10.73 & $0.947(0.862-0.983)$ & 11.02 & $0.984(0.956-0.996)$ & 15.19 \\
\hline Fmed & $0.881(0.678-0.965)$ & 6.37 & $0.884(0.701-0.964)$ & 8.82 & $0.902(0.747-0.969)$ & 8.84 & $0.943(0.786-0.986)$ & 5.12 \\
\hline Fmean & $0.813(0.517-0.941)$ & 5.93 & $0.930(0.818-0.978)$ & 4.54 & $0.898(0.737-0.968)$ & 5.57 & $0.952(0.829-0.987)$ & 3.85 \\
\hline Fmax & $0.802(0.466-0.942)$ & 16.25 & $0.725(0.220-0.925)$ & 10.71 & $0.744(0.339-0.919)$ & 17.17 & $0.928(0.729-0.982)$ & 6.80 \\
\hline
\end{tabular}

\footnotetext{
Cite this article: Juan Manuel Yáñez G, David Rodríguez R, Ricardo Mora C, Antonio G Ravelo G, Juan Ribas S, Juan José González B. Reliability of Mechanical and EMG Variables Assessed During Concentric Bench Press Exercise Against Different Submaximal Loads. Biomed J Sci\&Tech Res 9(3)-2018. BJSTR. MS.ID.001798. DOI: 10.26717/ BJSTR.2018.09.001798.
} 


\begin{tabular}{|c|c|c|c|c|c|c|c|c|}
\hline Fmax $_{0-300}$ & $0.775(0.393-0.934)$ & 19.22 & $0.544(-0.293-0.876)$ & 25.60 & $\begin{array}{c}0.530(-0.217- \\
0.852)\end{array}$ & 19.12 & $0.483(-0.956-0.869)$ & 15.44 \\
\hline FInsm5 & $0.902(0.736-0.971)$ & 25.28 & $0.846(0.602-0.952)$ & 23.80 & $0.875(0.676-0.961)$ & 29.20 & $0.954(0.825-0.988)$ & 28.62 \\
\hline
\end{tabular}

1RM: One-Repetition Maximum; ICC: Intraclass Correlation Coefficient; CI: Confidence Interval; CV: Coefficient of Variation; EMG: Electromyography; RMS: Root Mean Square; IEMG: Integrated EMG; Fmed: Median Frequency; Fmean: Mean Frequency; Fmax: Maximal Frequency; Finsm5: The New Spectral Parameter Proposed by Dimitrov.

\section{Discussion}

\section{Mechanical Variables}

The purpose of this study was to investigate intrasession reliability of several mechanical and EMG variables during the concentric phase of the BP exercise against different load magnitudes (50\%, 60\%, 70\% and 80\% 1RM). An important aspect of the present study was that, by monitoring repetition velocity and adjusting the actual loads to be lifted as determined from the loadvelocity relationship for the BP exercise [28], we made sure that all participants used a very similar relative load (\%1RM) in each testing session (Table 1). Thus, since all the participants performed the repetitions at a very similar MPV $\left( \pm 0.02 \mathrm{~m} \cdot \mathrm{s}^{-1}\right)$, the betweensubject variability was very low and consequently, the ICC was poor for this variable (Table 2). These results do not mean that the MPV is not a reliable variable $[2,3,32]$. The low relative reliability is a consequence of having taken the MPV as an independent variable for monitoring the training intensity. In fact, MPV showed a high absolute reliability for all load magnitudes used (CV: 1.40-2.90\%). Recent studies $[7,10]$ assessing the test-retest reproducibility of the whole load-velocity curve (20-100\% 1RM) and using a similar methodology (only concentric BP exercise executing each repetition at maximal intended velocity) have also shown high absolute reliability scores for the MPV (CV: 2-5\%), with greater $\mathrm{CV}$ values as the load magnitude increases. Similar results to MPV were found for MV and PV in the present (CV: 1.22-2.62\%; Table 2 ) and earlier studies (CV: 1.74-5.80\%) [8,10]. However, unlike the MPV and MV, the PV also showed a high relative reliability in all load magnitudes used (ICC: 0.927-0.957; Table 2). These results indicate that, although the MPV and MV were similar for all the participants, the PV resulted in a large between-subject variability (range: 1.47$1.78,1.30-1.55,0.98-1.23,0.82-1.01 \mathrm{~m} \cdot \mathrm{s}^{-1}$ for $50 \%, 60 \%, 70 \%$ and $80 \% 1 \mathrm{RM}$, respectively) suggesting that this variable is specific to each individual and also indicating that this variable allows us to discriminate between subjects with different performance levels.

Other mechanical variables such as PA, PP, PF and MPF also showed high reliability (ICC: 0.920-0.997; CV: 1.55-8.87\%; Table 2), with a clear tendency to lower ICC and greater CV values as load magnitude increased in both muscles assessed. In agreement with our results, Izquierdo et al. [9] found high absolute (CV: 4.7-7.9\%) and relative (ICC: $0.93-0.95)$ reliability for power output during the BP exercise against different submaximal loads (30\%, 45\%, 60\%, $70 \%$ and $80 \% 1 \mathrm{RM}$ ). In contrast, Young et al. [5] reported lower t

est-retest ICC scores (ICC: 0.85-0.92) than those obtained in the present study for PF, PP and PV. This could be because Young et al. [5] used a ballistic bench press throw involving a continuous eccentric-concentric motion without imposing a pause between both phases. It seems that this type of technical execution using a stretch-shortening cycle increases the biological within-subject variation of the mechanical variables assessed [7].

For the RFD variables, our results revealed that the interval width of the sampling window used in the calculation of MRFD influenced on the reliability values (Table 3). Although it has been speculated that the sampling window used for calculating MRFD during isometric contractions does not appreciably affect MRFD reliability [33], a recent study [24] showed that MRFD calculated using a $20 \mathrm{~ms}$ sampling window during an isometric midthigh clean pull resulted in higher ICC scores compared to other sampling window durations $(2,5,10,30$ and $50 \mathrm{~ms})$. In contrast, the results of the present study showed that MRFD exhibited high reliability scores (ICC: 0.901-0.976; CV: 7.20-13.79\%; Table 3) regardless of the sampling window durations and load magnitudes used, except for MRFD4-40 against 80\% 1RM, which showed a moderate absolute and relative reliability (Table 3 ). Therefore, our results suggest that in order to assess the MRFD during a concentric-only $\mathrm{BP}$, a sample window duration equal to or greater than $40 \mathrm{~ms}$ should be used to obtain more reliable measurements.

The time needed to reach MRFD showed moderate reliability in all load magnitudes used, with a tendency to show higher ICC and lower $\mathrm{CV}$ as the sampling windows duration for calculating MRFD increased (Table 3). Previous studies [34,35] using isometric knee extension have shown greater ICC (0.87-0.98) and lower CV (6.7$11.3 \%$ ) values for this variable compared to our study. Thus, these results seem to be in agreement with prior studies indicating that isometric tests allow a high level of control of the measurement and therefore, these tests have generally shown high reliability of RFD variables in both single and multi-joint test protocols compared to dynamic tests [25]. However, Chiu et al. [36] found high relative reliability (ICC: 0.91-0.97) for T_MRFD using a concentric-only jump squat against different loads (30\%, 50\%, 70\% 1RM in parallel squat), whereas the reliability was drastically lower (ICC: 0.19-0.58) during a rebound (eccentric-concentric) jump squat. Therefore, it appears that this variable could also reach high reliability levels during concentric dynamic activations.

Similar to T_MRFD, the time taken from the beginning of force production to the attainment of different fractions of the peak force also showed moderate to high ICC scores and CV values greater than $10 \%$ (Table 3). In addition, there was a slight tendency toward higher ICC and lower CV as the load magnitude and the percentage of the PF to be achieved increased (Table 3). Studies analyzing the reliability of this variable have shown contrasting results depending 
on the type of muscle contraction used [36,37]. In agreement with our results, a past study [36] revealed moderate to high reliability (ICC: $0.80-0.95$ ) for T20\%-T100\% (with 10\% increments) during a concentric-only jump test. However, when this variable was measured during isometric [37] or shortening-stretch cycle tests [36], the reliability was considerably lower. In addition, similar to the present study, both previous studies [36,37] showed that the reliability level was higher as the fraction of PF used increased, regardless of the type of muscle contraction used.

In a previous study, Haff et al. [24] reported that using predetermined time bands $(0-30,0-50,0-90,0-100,0-150,0-200$, $0-250 \mathrm{~ms}$ ) for the calculation of RFD during the isometric midthigh clean pull resulted in a very high degree of reliability (ICC $>0.9$ and $\mathrm{CV}<4 \%$ ). Additionally, this study [24] showed that the reliability scores for predetermined time bands were higher compared to MRFD (regardless of the sampling windows used). In contrast, our results showed that (a) the absolute and relative RFD calculated using predetermined time bands resulted in moderate relative and poor absolute reliability scores (ICC: $0.550-0.918$; CV: $18.36-$ $52.68 \%$; Table 3); and (b) the reliability for MRFD was greater compared to RFD in different time bands. Discrepancies with Haff et al. [24] could be due to differences in both the type of contraction (isometric vs. only concentric) and the type of exercise (midthigh clean pull vs. BP). However, previous studies using isometric knee extension [26] and isometric plantar flexion [21] revealed similar results to those found in the present study. Moreover, in agreement with other studies $[21,26,38]$, the use of shorter time intervals in the calculations of RFD appears to increase variability and reduce absolute and relative reliability. These lower reliability scores noted in the initial contraction phase could be the result of a low signal-to-noise ratio. Therefore, although early RFD ( $<50 \mathrm{~ms})$ seems to be relevant for successful performance of various functional movements $[23,39]$, caution should be applied when interpreting changes in rapid force capacity obtained within the initial $25-50 \mathrm{~ms}$ time interval relative to contraction onset following longitudinal training protocols $[13,26]$.

\section{EMG Variables}

Generally, all EMG variables assessed showed moderate to high reliability for all load magnitudes, although the variable FInsm5 resulted in very poor absolute reliability (CV > 20\%). Similar results have been found for EMG registrations during static $[14,40]$ and dynamic $[15,16,20]$ contractions, although none of the previous studies were conducted using the BP exercise. On the other hand, comparisons between EMG variables revealed higher ICC scores for RMS and iEMG compared to Fmed, Fmean, Fmax and FInsm5 (Tables 4,5). These results are consistent with those shown in previous studies $[14,15]$. However, similar to previous observations $[20,41,42]$, our results also revealed that Fmed and Fmean showed lower CV values than RMS and IEMG. Thus, considering that an ICC score is mainly dependent on the between-subject variability in the data (heterogeneity of the subjects), and the CV is related to the variability of individual scores on a test and is unaffected by the range of measurements $[2,32]$, these results indicate greater consistency and intra-subject stability for Fmed and Fmean compared to RMS and iEMG. Therefore, our results suggest that frequency domain EMG variables may be more appropriate than EMG variables related to amplitude or time distribution (RMS and iEMG) for analyzing changes in neuromuscular performance.

On the other hand, our results showed no relevant effect of load magnitude on reliability of EMG variables in any of the muscles assessed (Tables 4,5). The absolute and relative reliability scores were very similar for all four load magnitudes used. A recent study [11] analyzed the reproducibility of RMS, iEMG and Fmed in four muscles (gluteus maximus, biceps femoris, vastus lateralis and vastus medialis) during the squat exercise and indicated that the EMG variables differed slightly in reliability across the various load magnitudes (70\%, 80\% and 90\% 1RM) of resistance exercise used. However, results of this study [11] seem to indicated that the impact of load magnitude on reliability of EMG variables is also dependent on the muscle assessed. Thus, it appears that further investigations are needed in order to know the effect of load on the reliability of EMG variables.

\section{Conclusion}

The main findings of the present study were:

a) All mechanical variables showed a high reliability, except for T_MRFD, absolute and relative RFD in different time bands and the time taken to the attainment of $25 \%, 50 \%$ and $75 \%$ of peak force;

b) RMS, iEMG, Fmed and Fmean were the EMG variables with the highest reliability scores for all load magnitudes and both muscles assessed;

c) The longer the time interval used to quantify the RFD, the higher the reliability level;

d) Reliability of mechanical variables was affected by the load magnitude used, with generally lower reliability scores as load magnitude increased;

e) Reliability of EMG variables was not affected by the load magnitude used; and

f) Reliability of EMG variables was similar for both muscles assessed.

\section{Practical Applications}

Based on the results of the present study, several critical implications may be derived from this investigation to optimize the assessment of neuromuscular performance during upper limb movements. Firstly, variables related to force, velocity and power output showed high reliability. Secondly, the method used to quantify the RFD also impacts the reliability of the measurement. Our results indicate that MRFD is the variable with the highest reliability scores. Thus, this variable should be preferably used when analyzing the force-time curve during a concentric-only BP exercise. Furthermore, if predetermined time-interval bands are used to quantify RFD during this exercise, it is recommended that strength and conditioning professionals preferentially use time bands greater than $50 \mathrm{~ms}$. On the other hand, our results 
also showed that the reliability of several mechanical variables is affected by the load magnitude used. Thus, coaches should exert caution when using very high loads ( $>80 \% 1 \mathrm{RM})$ to assess strength performance in athletes, since the reliability of the measurement decreases as the load used increases. Finally, if EMG is measured to assess neuromuscular performance, the RMS, iEMG, Fmed and Fmean should preferably be used.

\section{Acknowledgement}

We appreciate the time and effort of the participants who took part in this study.

\section{References}

1. Abernethy P, Wilson G, Logan P (1995) Strength and power assessment. Issues, controversies and challenges. Sports Med 19(6): 401-417.

2. Atkinson G, Nevill AM (1998) Statistical methods for assessing measurement error (reliability) in variables relevant to sports medicine. Sports Med 26(4): 217-238.

3. Hopkins WG (2000) Measures of reliability in sports medicine and science. Sports Med 30(1): 1-15.

4. Padulo J, Laffaye G, Chaouachi A, Chamari K (2015) Bench press exercise: The key points. J Sports Med Phys Fitness 55(6): 604-608.

5. Young KP, Haff GG, Newton RU, Sheppard JM (2014) Reliability of a novel testing protocol to assess upper-body strength qualities in elite athletes. Int J Sports Physiol Perform 9(5): 871-875.

6. Pryor JF, Wilson GJ, Murphy AJ (1994) The effectiveness of eccentric, concentric and isometric rate of force development test. J Hum Mov Stud 27(4): 153-172.

7. Pallares JG, Sanchez-Medina L, Perez CE, De La Cruz-Sanchez E, MoraRodriguez R (2014) Imposing a pause between the eccentric and concentric phases increases the reliability of isoinertial strength assessments. J Sports Sci 32(12): 1165-1175.

8. Stock MS, Beck TW, DeFreitas JM, Dillon MA (2011) Test-retest reliability of barbell velocity during the free-weight bench-press exercise. J Strength Cond Res 25(1): 171-177.

9. Izquierdo M, Hakkinen K, Gonzalez-Badillo JJ, Ibanez J, Gorostiaga EM (2002) Effects of long-term training specificity on maximal strength and power of the upper and lower extremities in athletes from different sports. Eur J Appl Physiol 87(3): 264-271.

10. Garcia-Ramos A, Pestana-Melero FL, Perez-Castilla A, Rojas FJ, Gregory Haff G (2018) Mean Velocity vs. Mean Propulsive Velocity vs. Peak Velocity: Which Variable Determines Bench Press Relative Load With Higher Reliability? J Strength Cond Res 32(5): 1273-1279.

11. Scott BR, Slattery KM, Sculley DV, Lockie RG, Dascombe BJ (2014) Reliability of telemetric electromyography and near-infrared spectroscopy during high-intensity resistance exercise. J Electromyogr Kinesiol 24(5): 722-730.

12. Tabard-Fougere A, Rose-Dulcina K, Pittet V, Dayer R, Vuillerme N, et al. (2017) EMG normalization method based on grade 3 of manual muscle testing: Within- and between-day reliability of normalization tasks and application to gait analysis. Gait Posture 60: 6-12.

13. Sleivert GG, Wenger HA (1994) Reliability of measuring isometric and isokinetic peak torque, rate of torque development, integrated electromyography and tibial nerve conduction velocity. Arch Phys Med Rehabil 75(12): 1315-1321.

14. Viitasalo JH, Komi PV (1975) Signal characteristics of EMG with special reference to reproducibility of measurements. Acta Physiol Scand 93(4): 531-539.

15. Larsson B, Mansson B, Karlberg C, Syvertsson P, Elert J, et al. (1999) Reproducibility of surface EMG variables and peak torque during three sets of ten dynamic contractions. J Electromyogr Kinesiol 9(5): 351-357.
16. Larsson B, Karlberg C, Elert J, Gerdle B (1999) Reproducibility of surface EMG during dynamic shoulder forward flexions: A study of clinically healthy subjects. Clin Physiol 19(5): 433-439.

17. Fauth ML, Petushek EJ, Feldmann CR, Hsu BE, Garceau LR, et al. (2010) Reliability of surface electromyography during maximal voluntary isometric contractions, jump landings, and cutting. J Strength Cond Res 24(4): 1131-1137.

18. Goodwin PC, Koorts K, Mack R, Mai S, Morrissey MC, et al. (1999) Reliability of leg muscle electromyography in vertical jumping. Eur J Appl Physiol Occup Physiol 79(4): 374-378.

19. Gollhofer A, Horstmann GA, Schmidtbleicher D, Schonthal D (1990) Reproducibility of electromyographic patterns in stretch-shortening type contractions. Eur J Appl Physiol Occup Physiol 60(1): 7-14.

20. Carius D, Kugler P, Kuhwald HM, Wollny R (2015) Absolute and relative intrasession reliability of surface EMG variables for voluntary precise forearm movements. J Electromyogr Kinesiol 25(6): 860-869.

21. Thompson BJ, Ryan ED, Herda TJ, Costa PB, Walter AA, et al. (2012) Consistency of rapid muscle force characteristics: influence of muscle contraction onset detection methodology. J Electromyogr Kinesiol 22(6): 893-900.

22. Folland JP, Buckthorpe MW, Hannah R (2014) Human capacity for explosive force production: Neural and contractile determinants. Scand J Med Sci Sports 24(6): 894-906.

23. Tillin NA, Jimenez-Reyes P, Pain MT, Folland JP (2010) Neuromuscular performance of explosive power athletes versus untrained individuals. Med Sci Sports Exerc 42(4): 781-790.

24. Haff GG, Ruben RP, Lider J, Twine C, Cormie P (2015) A comparison of methods for determining the rate of force development during isometric midthigh clean pulls. J Strength Cond Res 29(2): 386-395.

25. Rodriguez-Rosell D, Pareja-Blanco F, Aagaard P, Gonzalez-Badillo JJ (2018) Physiological and methodological aspects of rate of force development assessment in human skeletal muscle. Clin Physiol Funct Imaging 38(5): 743-762.

26. Buckthorpe MW, Hannah R, Pain TG, Folland JP (2012) Reliability of neuromuscular measurements during explosive isometric contractions, with special reference to electromyography normalization techniques. Muscle Nerve 46(4): 566-576.

27. Moir G, Sanders R, Button C, Glaister M (2005) The influence of familiarization on the reliability of force variables measured during unloaded and loaded vertical jumps. J Strength Cond Res 19(1): 140145 .

28. Gonzalez-Badillo JJ, Sanchez-Medina L (2010) Movement velocity as a measure of loading intensity in resistance training. Int J Sports Med 31(5): 347-352.

29. Hermens HJ, Freriks B, Disselhorst-Klug C, Rau G (2000) Development of recommendations for SEMG sensors and sensor placement procedures. J Electromyogr Kinesiol 10(5): 361-374.

30. Aagaard P, Simonsen EB, Andersen JL, Magnusson P, Dyhre-Poulsen P (2002) Increased rate of force development and neural drive of human skeletal muscle following resistance training. J Appl Physiol 93(4): $1318-1326$

31. Dimitrov GV, Arabadzhiev TI, Mileva KN, Bowtell JL, Crichton N, et al. (2006) Muscle fatigue during dynamic contractions assessed by new spectral indices. Med Sci Sports Exerc 38(11): 1971-1979.

32. Weir JP (2005) Quantifying test-retest reliability using the intraclass correlation coefficient and the SEM. J Strength Cond Res 19(1): 231-240.

33. Wilson GJ, Murphy AJ (1996) The use of isometric tests of muscular function in athletic assessment. Sports Med 22(1): 19-37.

34. de Ruiter CJ, de Korte A, Schreven S, de Haan A (2010) Leg dominancy in relation to fast isometric torque production and squat jump height. Eur $\mathrm{J}$ Appl Physiol 108(2): 247-255. 
35. de Ruiter CJ, Vermeulen G, Toussaint HM, de Haan A (2007) Isometric knee-extensor torque development and jump height in volleyball players. Med Sci Sports Exerc 39(8): 1336-1346.

36. Chiu LZ, Schilling BK, Fry AC, Weiss LW (2004) Measurement of resistance exercise force expression. J Appl Biomech 20(2): 204-212.

37. Going SB, Massey BH, Hoshizaki TB, Lohman TG (1987) Maximal voluntary static force production characteristics of skeletal muscle in children 8-11 years of age. Res Q Exerc Sport 58(2): 115-123.

38. Tillin NA, Pain MT, Folland JP (2011) Short-term unilateral resistance training affects the agonist-antagonist but not the force-agonist activation relationship. Muscle Nerve 43(3): 375-384.

39. de Ruiter CJ, Van Leeuwen D, Heijblom A, Bobbert MF, de Haan A (2006) Fast unilateral isometric knee extension torque development and bilateral jump height. Med Sci Sports Exerc 38(10): 1843-1852.

\section{ISSN: 2574-1241}

DOI: $10.26717 / B J S T R .2018 .09 .001798$

David Rodríguez Rosell. Biomed J Sci \& Tech Res

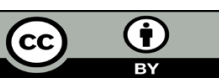

This work is licensed under Creative Commons Attribution 4.0 License

Submission Link: https://biomedres.us/submit-manuscript.php
40. Pincivero DM, Green RC, Mark JD, Campy RM (2000) Gender and muscle differences in EMG amplitude and median frequency and variability during maximal voluntary contractions of the quadriceps femoris. J Electromyogr Kinesiol 10(3): 189-196.

41. Viitasalo JT, Saukkonen S, Komi PV (1980) Reproducibility of measurements of selected neuromuscular performance variables in man. Electromyogr Clin Neurophysiol 20(6): 487-501.

42. Larsson B, Karlsson S, Eriksson M, Gerdle B (2003) Test-retest reliability of EMG and peak torque during repetitive maximum concentric knee extensions. J Electromyogr Kinesiol 13(3): 281-287.

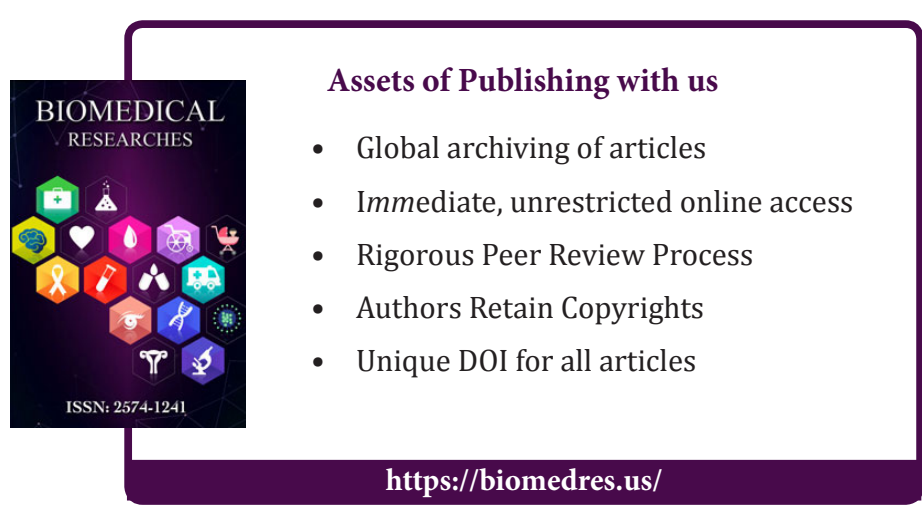

Cite this article: Juan Manuel Yáñez G, David Rodríguez R, Ricardo Mora C, Antonio G Ravelo G, Juan Ribas S, Juan José González B. Reliability of Mechanical and EMG Variables Assessed During Concentric Bench Press Exercise Against Different Submaximal Loads. Biomed J Sci\&Tech 